Pacific Journal of Mathematics

A COHOMOLOGICAL INTERPRETATION OF BRAVER 


\title{
A COHOMOLOGICAL INTERPRETATION OF BRAUER GROUPS OF RINGS
}

\author{
RAYMOND T. HOOBLER \\ Dedicated to Gerhard Hochschild on the occasion of his 65th birthday
}

Quillen's proof of the Serre conjecture introduced a new tool for passing from local to global results on affine schemes. We use this to prove the theorem below characterizing the image of the injection $i: B r(X) \rightarrow H^{2}\left(X_{c t}, G_{m}\right)$ when $X=$ Spec $A$, is a regular scheme. A result of M. Artin then allows us to conclude that $B r(X) \cong H^{2}\left(X_{e t}, G_{m}\right)$ if $X=\operatorname{Spec} A$ is a smooth, affine scheme over a field. For such rings, this proves the Auslander Goldman conjecture [2], $\operatorname{Br}(A)=\cap \operatorname{Br}\left(A_{\psi}\right)$, $\mathfrak{p} \in P(A)$, the set of height one primes of $A$.

We begin with following theorem.

Theorem. Let $X=\operatorname{Spec} A$ be a regular scheme. If $c \in H^{2}\left(X_{e t}, G_{m}\right)$ and $c_{y}=i\left(\left[\Lambda_{y}\right]\right)$ in $H^{2}\left(\operatorname{Spec}\left(A_{\mathrm{w}_{y}}\right)_{e}, G_{m}\right)$ for all closed points $y \in X$, then $c=i([\Lambda])$.

Proof. If $f \in A$, let $c_{f}$ denote the restriction of $c$ to

$$
H^{2}\left(\operatorname{Spec}\left(A_{f}\right)_{e t}, G_{m}\right) \text {. }
$$

Let $S=\left\{f \in A \mid c_{f}=i([\Lambda])\right.$ for some Azumaya algebra $\Lambda$ over $\left.A_{f}\right\}$. We will show that $S$ is an ideal. Then $S=A$ since the hypothesis on $c$ prevents $S$ from being contained in any maximal ideal of $A$.

Suppose $f_{1}, f_{2} \in S$ and $f \in A f_{1}+A f_{2}$. Then $\operatorname{Spec}\left(A_{f}\right)=D_{f_{1}} \cup D_{f_{2}}$ where $D_{f_{i}}=\operatorname{Spec}\left(A_{f f_{1}}\right)$. Hence we may assume $A_{f}=A$ and $\operatorname{Spec}(A)$ is covered by $D_{f_{1}} \cup D_{f_{2}}$. Let $\Lambda_{1}, \Lambda_{2}$ be Azumaya algebras over $A_{f_{1}}, A_{f_{2}}$ with $i\left(\left[\Lambda_{1}\right]\right)=c_{f_{1}}$ and $i\left(\left[\Lambda_{2}\right]\right)=c_{f_{2}}$. Since $i$ is injective, $\left[\Lambda_{1 f_{2}}\right]=\left[\Lambda_{2 f_{1}}\right]$; that is, there are locally free coherent $A_{f_{1} f_{2}}$ modules $P_{1}, P_{2}$ such that $A_{1 f_{2}} \otimes \operatorname{End}\left(P_{1}\right) \cong \Lambda_{2 f_{2}} \otimes \operatorname{End}\left(P_{2}\right)$. Since $K^{0}\left(A_{f_{i}}\right) \rightarrow K^{0}\left(A_{f_{1} f_{2}}\right)$ is onto $(A$ is regular) [3] and we may assume the rank of $P_{i}$ is large, there are locally free coherent $A_{f_{i}}$ modules $Q_{i}$ such that $Q_{i f_{i}} \cong P_{i}$ [3, Chapter IX, 4.1]. Replacing $\Lambda_{i}$ by $\Lambda_{i} \otimes \operatorname{End}\left(Q_{i}\right)$, we may assume that $\Lambda_{1 f_{2}} \cong \Lambda_{2 f_{1}}$. Using this patching isomorphism we produce an algebra $\Lambda$ with $\Lambda_{f_{1}} \cong \Lambda_{1}, \Lambda_{f_{2}} \cong \Lambda_{2}$. Since $H^{2}\left(X_{e t}, G_{m}\right) \rightarrow H^{2}\left(D_{f_{i} e t}, G_{m}\right)$ is a monomorphism, $c=i([\Lambda])$.

CoRollary 1. Let $X=\operatorname{Spec}(A)$ be a smooth $k$ scheme where $k$ is a field. Then $\operatorname{Br}(X) \cong H^{2}\left(X_{e t}, G_{m}\right)$.

Proof. Since $X$ is regular, $H^{2}\left(X_{e t}, G_{m}\right)$ is torsion [4]. If $c \in$ 
$H^{2}\left(X_{e t}, G_{m}\right)$ has order $n$ and $n$ is relatively prime to the characteristic of $k$, then the Kummer sequence

$$
o \longrightarrow \mu_{n} \longrightarrow G_{m} \longrightarrow G_{m} \longrightarrow 0
$$

shows that $c$ is in the image of $H^{2}\left(X_{e t}, \mu_{u}\right)$. Now the existence of good neighborhoods [1] on $\bar{X}=X \bigotimes_{k} \bar{k}, \bar{k}=$ algebraic closure of $k$, shows that elements of $H^{2}\left(\bar{X}_{e t}, \mu_{n}\right)$ are locolly isotrivial, i.e., there is a Zariski covering $\left\{U_{i}\right\}$ of $\bar{X}$ and a finite, etale covering space $V_{i} \rightarrow$ $U_{i}$ which splits elements of $H^{2}\left(X_{e t}, \mu_{n}\right)$. Consequently $X$ has a Zariski open covering $\left\{U_{\alpha}\right\}$ and finite, flat coverings $\pi_{\alpha}: W_{\alpha} \rightarrow U_{\alpha}$ such that $\pi_{\alpha}^{*}\left(\left.c\right|_{U_{\alpha}}\right)=0$. Hence by the criterion in [6], $\left.c\right|_{U_{\alpha}}$ is in the image of $\operatorname{Br}\left(U_{\alpha}\right)$ and so by the theorem $c$ is in the image of $\operatorname{Br}(X)$. If $c$ has order $p^{n}, p=$ char $k$, then we know that $F_{X}^{n^{*}}(c)=0$ where $F_{X}$ is the Frobenius map. Since it defines a finite flat covering of $X$, the same criterion shows that $c$ is in the image of $B r(X)$ (see [7] where this argument is given in more detail.).

CoRollary 2. Let $A$ be an algebra of finite type over a field $k$ such that $A \otimes \vec{k}$ is regular, i.e., Spec $A$ is smooth over $k$. Then

$$
\operatorname{Br}(A)=\cap \operatorname{Br}\left(A_{\mathfrak{p}}\right), \mathfrak{p} \in P(A)=\{\mathfrak{p} / \text { height } \mathfrak{p}=1\} .
$$

Proof. We will use induction on $n=\operatorname{dim} A$. If $n=0,1$, or 2 , the result was proven in [2, 4]. Since $A$ is a regular $\operatorname{ring}, \operatorname{Br}(A) \subset \cap$ $\operatorname{Br}\left(A_{\mathfrak{p}}\right), \mathfrak{p} \in P(A)$. Hence the argument of the theorem shows that

$$
S=\left\{f \in A / B r\left(A_{f}\right)=\cap \operatorname{Br}\left(A_{\mathfrak{p}}\right), \mathfrak{p} \in P\left(A_{f}\right)\right\}
$$

is an ideal in $A$ and so is either $A$ or is contained in a maximal ideal of $A$. Hence we may assume $A$ is a local ring of dimension greater than 2 .

Let $c \in \cap \operatorname{Br}\left(A_{\mathfrak{p}}\right), \mathfrak{p} \in P(A)$, and $X=$ Spec $A$ and $U$ be the punctured spectrum. Since $\operatorname{Br}\left(A_{\mathfrak{p}}\right)=H^{2}\left(\operatorname{Spec}\left(A_{\mathfrak{p}}\right)_{e t}, G_{m}\right)$, there is a cohomology class $c_{1} \in \cap H^{2}\left(\operatorname{Spec}\left(A_{\mathfrak{p}}\right)_{e t}, G_{m}\right) \subseteq H^{2}\left(\operatorname{Spec}(K)_{e t}, G_{m}\right), \mathfrak{p} \in P(A)$, with $c_{1}=$ $i(c)$ where $K$ is the quotient field of $A$. Now the Mayer-Vietoris sequence, which may be viewed as the Cech spectral sequence for the covering $\left\{U_{1}, U_{2}\right\}$ of $U_{1} \cup U_{2}$, and the induction hypothesis show that there is a $c^{\prime} \in H^{2}\left(U_{e t}, G_{m}\right)$ whose restriction to $H^{2}\left(\operatorname{Spec}\left(A_{e t}, G_{m}\right)\right.$ is $i(c)$. Suppose $c^{\prime}$ is of order $n$ where $(n, \operatorname{char} k)=1$. Then the Kummer sequence shows that there is a cohomology class in $H^{2}\left(U_{e t}, \mu_{n}\right)$ whose image in $H^{2}\left(U_{e t}, G_{m}\right)$ is $c^{\prime}$. But $H^{2}\left(X_{e t}, \mu_{n}\right)=H^{2}\left(U_{e t}, \mu_{n}\right)$ by relative cohomological purity [1, Expose XVI] and so there is a cohomology class $c^{\prime \prime}$ in $H^{2}\left(X_{e t}, G_{m}\right)$ whose restriction to $U$ is $c^{\prime}$. By the first corollary $c^{\prime \prime}$ is in the image of $\operatorname{Br}(X)$ as desired.

If $n=p^{m}, p=\operatorname{char}(k)$, the same argument will work if we can 
show that $e^{\prime}$ is in the image of $H^{2}\left(X_{e t}, G_{m}\right)$. We will then be done since $c^{\prime}=c_{1}^{\prime}+c_{2}^{\prime}$ where the order of $c_{1}^{\prime}=p^{m}$ and the order of $c_{2}^{\prime}$ is prime to $p$. The obstruction to $c^{\prime}$ being in the image of $H^{2}\left(X_{e t}, G_{m}\right)$ lies in the local cohomology group $H_{P}^{3}\left(X_{e t}, G_{m}\right)$ where $P$ is the closed point of $X$. Moreover since $F_{X^{*}}^{m}\left(c^{\prime}\right)=p^{m} c^{\prime}=0$ where $F_{X}: X \rightarrow X$ is the purely inseparable Galois covering defined by the Frobenius map, the obstruction lies in the kernel of $F_{X}^{m^{*}}: H_{P}^{3}\left(X_{e t}, G_{m}\right) \rightarrow H_{P}^{3}\left(X_{e t}, G_{m}\right)$.

We have an exact sequence of sheaves on $X_{e t}$ [7]

$$
0 \longrightarrow G_{m} \stackrel{j}{\longrightarrow} F_{X^{*}} G_{m} \longrightarrow \mathscr{F}_{X}^{1} \longrightarrow \Omega_{X}^{1} \longrightarrow 0
$$

where $\mathcal{\Sigma}_{X}^{-1}$ and $\Omega_{X}^{1}$ are free $A$-modules ( $A$ is smooth and local) whose definition is unimportant. If $C$ denotes the cokernel of $j$, then $H_{P}^{2}\left(X_{e t}, C\right)$ is trapped between $H_{P}^{1}\left(X_{e t}, \Omega_{X}^{1}\right)$ and $H_{P}^{2}\left(X_{e t}, \mathscr{K}_{X}^{1}\right)$. Since $\Omega_{X}^{1}$ and $\mathscr{X}_{X^{1}}^{1}$ are coherent sheaves, their local cohomology in the etale and Zariski topology coincide and hence vanish because $H_{|\mathrm{m}|}^{i}(\operatorname{Spec}(A), A)=0$ if $A$ is regular and $i<\operatorname{dim} A$. Since $\operatorname{dim} A>2$, we conclude that

$$
F_{\lambda}^{*}: H_{P}^{3}\left(X_{e t}, G_{m}\right) \longrightarrow H_{P}^{3}\left(X_{e t}, F_{X^{*}} G_{m}\right)=H_{P}^{3}\left(X_{e t}, G_{m}\right)
$$

is injective and so $c^{\prime}$ is in the image of $H^{2}\left(X_{e t}, G_{m}\right)$.

COROLlaRy 3. Let $\pi: X \rightarrow Y$ be a proper, smooth morphism of fibre dimension one where $X$ is a smooth scheme over a field. Then $R^{2} \pi_{*} G_{m}=0$.

Proof. We may assume that $Y$ is a strictly local $k$-scheme, $k$ a field, and we must show that $H^{2}\left(X_{e t}, G_{m}\right)=0$. Since $\pi$ has fibre dimension one and is proper, $X$ is a union of two affine schemes which are limits of smooth $k$-schemes. Consequently $H^{2}\left(X_{e t}, G_{m}\right)=$ $B r(X)$. But $B r\left(\pi^{-1}(y)\right)=0$ by Tsen's theorem. Thus Artin approximation may be used as in [5] to lift a trivialization of an Azumaya algebra on the fibre to a trivialization of the algebra on $X$.

\section{REFERENCES}

1. M. Artin and A. Grothendieck, Theorie des Topos et Cohomologie Etale des Schemas, III (S. G. A. A.), Lecture Notes in Mathematics, 305 (1973), Springer Verlag, 64-78.

2. M. Auslander and O. Goldman, The Brauer group of a commutative ring, Transactions Amer. Math. Soc., 97 (1960), 367-409.

3. H. Bass, Algebraic K-Theory, Mathematics Lecture Notes Series (1968), W. A. Benjamin, New York.

4. A. Grothendieck, Le Groupe de Brauer II, Dix Exposes sur la Cohomologie des Schemas, North Holland, Amsterdam, (1969), 66-87.

5. - Le Groupe de Brauer III, Dix Exposes sur la Cohomologie des Schemas, North Holland, Amsterdam, 1969. 
6. R. Hoobler, Brauer groups of Abelian schemes, Annales Scientifique de l'E.N.S. (4), 5 (1972), 45-70.

7. - Cohomology of purely inseparable Galois coverings, Journal fur die Reine und angewandte Mathematik, 266 (1974), 183-199.

Received March 21, 1979. This work was partially supported by NSF Grant No. MSC 76-06768 and a RFCUNY FRAP Award No. 11670.

\section{City COLlege}

CUNY

NEW YoRK, NY 10031 


\section{PACIFIC JOURNAL OF MATHEMATICS}

\section{EDITORS}

DONALD BABBITT (Managing Editor)

University of Galifornia

Los Angeles, California 90024

HUGo RossI

University of Utah

Salt Lake City, UT 84112

C. C. MOORE AND ANDREW OGG

University of California

Berkeley, CA 94720
J. DUGUNDJI

Department of Mathematics University of Southern California Los Angeles, California 90007

R. FINN AND J. MILGRAM Stanford University Stanford, California 94305

\section{ASSOCIATE EDITORS}

E. F. BECKENBACH

B. H. NEUMANN

F. WOLF

K. YosHIDA

\section{SUPPORTING INSTITUTIONS}

UNIVERSITY OF BRITISH COLUMBIA CALIFORNIA INSTITUTE OF TECHNOLOGY UNIVERSITY OF CALIFORNIA MONTANA STATE UNIVERSITY UNIVERSITY OF NEVADA, RENO NEW MEXICO STATE UNIVERSITY OREGON STATE UNIVERSITY UNIVERSITY OF OREGON
UNIVERSITY OF SOUTHERN CALIFONIA STANFORD UNIVERSITY UNIVERSITY OF HAWAII UNIVERSITY OF TOKYO UNIVERSITY OF UTAH WASHINGTON STATE UNIVERSITY UNIVERSITY OF WASHINGTON 


\section{Pacific Journal of Mathematics}

\section{Vol. 86, No. $1 \quad$ November, 1980}

Gert Einar Torsten Almkvist, Invariants, mostly old ones .............. 1

Hyman Bass, Groups of integral representation type ................ 15

A. Białynicki-Birula, On action of SL(2) on complete algebraic

varieties........................................

Frederick Paul Greenleaf and Martin Allen Moskowitz, Groups of

automorphisms of Lie groups: density properties, bounded orbits, and

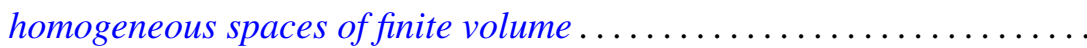

Raymond Taylor Hoobler, A cohomological interpretation of Brauer groups

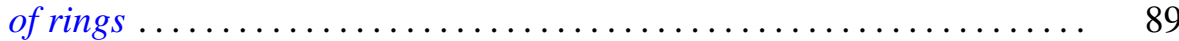

Irving Kaplansky, Superalgebras ........................ 93

Jerrold Lewis Kleinstein and Alex I. Rosenberg, Succinct and

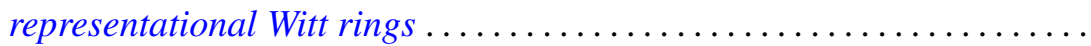

E. R. Kolchin, On universal extensions of differential fields ............ 139

Andy R. Magid, Analytic subgroups of affine algebraic groups. II ....... 145

Calvin Cooper Moore, The Mautner phenomenon for general unitary

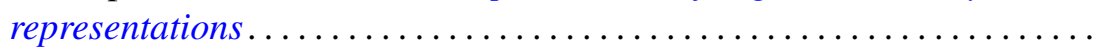

George Daniel Mostow, On a remarkable class of polyhedra in complex hyperbolic space ................................ 171

Brian Lee Peterson, Extensions of pro-affine algebraic groups. II . . . . . . 277

John Henry Reinoehl, Lie algebras and affine algebraic groups......... 287

Maxwell Alexander Rosenlicht, Differential valuations .

John Brendan Sullivan, The second Lie algebra cohomology group and Weyl modules..................................... 321

Moss Eisenberg Sweedler, Right derivations and right differential

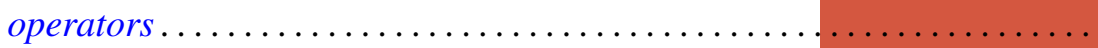

Bostwick Frampton Wyman, Time varying linear discrete-time systems. II. Duality.................................. 